\title{
Excimer Fluorescence of Poly(polyoxytetramethylene-glycol naphthalene-2,6-dicarboxylate)
}

\author{
Ti CAO, Xingkang ZHANG, and Renyuan QIAN* \\ Institute of Chemistry, Academia Sinica, Beijing 100080, China
}

(Received December 4, 1988)

\begin{abstract}
Time resolved emission spectra and fluorescence decay for poly(polyoxytetramethylene-glycol naphthalene-2,6-dicarboxylate) (PTMN) and its model compound dimethyl naphthalene-2,6-dicarboxylate (DMN) have been studied by time correlated single photon counting technique both in $\mathrm{CHCl}_{3}$ solution and in the neat polymer. The existence of intrachain excimer interaction between remote chromophores in the PTMN chain was demonstrated in dilute solutions. Inter-chain excimer interaction was demonstrated in concentrated solutions in $\mathrm{CHCl}_{3}$ for both PTMN and DMN. For a neat PTMN polymer sample which had been laid aside for a long time direct excitation of the excimer fluorescence from stacked pairs of the chromophores of the chain has been observed. A monomer fluorescence peak at $\lambda 400 \mathrm{~nm}$ was also observed in the early gated emission spectra when excited by $\lambda 381 \mathrm{~nm}$ radiation at room temperature. This is probably originated from hot band excitation. Evidence for the excimer dissociation has been obtained in concentrated solutions of both DMN and PTMN and in the neat polymer. The ratios of the pre-exponential coefficients of the shorter lifetime term to that of the longer lifetime term in the excimer fluorescence decay were found to be $-1 / 3,-1 / 2$, and $-1 / 5$ for the concentrated solution of DMN, the concentrated solution of PTMN and the neat polymer respectively. This result is different from the value -1 to be expected from Birk's diffusion controlled mechanism.
\end{abstract}

KEY WORDS Excimer Fluorescence / Preformed Excimer Site / TimeResolved Fluorescence Spectra / Poly(ether-ester) / Fluorescence Decay /

Since Yanari et al. ${ }^{1}$ found excimer fluorescence in polystyrene solution, excimer interaction in polymers containing aromatic chromophores has been of wide interest. Most authors have dealt with intra-chain excimer formation between adjacent aromatic chromophores of aromatic vinyl polymers ${ }^{2-7}$ and copolymers $^{8-13}$ in dilute solutions. Polymers with aromatic rings in the main chain would be much more revealing so far as the inter-chain interaction is concerned. Intra-chain excimer fluorescence of polymers containing naphthalene groups in the main chain separated by longer flexible spacers has been observed in dilute solutions. ${ }^{14,15}$ In our previous work ${ }^{16}$ the excimer fluorescence in three poly(polyehter glycol arylenedicarboxylate)s, in which the arylenedicarboxyloyl groups in the main chain are separated by polyether soft segments, demonstrated the existence of intra-chain excimer interaction between non-adjacent chromophores and found, in addition, the existence of a long wavelength shoulder in the excitation spectrum of a neat poly(polyoxytetramethylene-glycol naphthalene-2,6-dicarboxylate) (PTMN) sample which had been laid aside at room temperature for a long time. It was later found that this polymer could be crystallized in the form of spherulites during slow evaporation of the solution

\footnotetext{
* To whom correspondence should be addressed.
} 
above $40 \mathrm{C}$ or from the neat polymer. ${ }^{17}$ This shows that the naphthalene dicarboxyloyl groups in PTMN are stacking in the concentrated solution or in the neat polymer to form the crystalline phase. It is reasonable to assume that the origin of the long wavelength shoulder in the excitation spectrum of PTMN is the absorption of stacked naphthalene dicarboxyloyl groups.

In this paper the existence of intra-chain excimer interaction between remote chromophores in the main chain and the existence of direct excitation of excimer fluorescence from stacked aromatic ring chromophores as the preformed excimer sites in the neat polymer are demonstrated by transient fluorescence technique.

\section{EXPERIMENTAL}

\section{The Samples}

Dimethyl naphthalene-2,6-dicarboxylate (DMN) was purified by recrystallization twice from xylene, $\mathrm{mp} 188.7-189.2^{\circ} \mathrm{C}$. The polymer PTMN was prepared from trans-esterification polymerization of DMN and poly(oxytetramethylene)-glycol of $M_{n} \times 1.7 \cdot 10^{3}$ at 210 $230^{\circ} \mathrm{C}$ under argon atmosphere for $1.5 \mathrm{~h}$, using a methanol solution of $\mathrm{MgAc}_{2}$ and Ti$(\mathrm{OBu})_{4}$ as catalyst. ${ }^{16}$ Heating at $230^{\circ} \mathrm{C}$ was continued under a vacuum of $2 \times 10^{-2}$ torr for another hour. The polymer was purified by repeated precipitation five times from a $3-5 \mathrm{~g} \mathrm{dl}^{-1}$ solution in $\mathrm{CHCl}_{3}$ by adding $\mathrm{CH}_{3}$ $\mathrm{OH}$ as precipitant and then vacuum dried at $60^{\circ} \mathrm{C}$ to constant weight. There was no impurity of low molar mass in the polymer as shown in its size exclusion chromatogram using THF as eluent. The molar mass distribution of the polymer was found to be rather narrow and $\bar{M}_{n}=1.79 \times 10^{4}$ as determined by VPO from $\mathrm{CHCl}_{3}$ solution.

\section{Transient Fluorescence Measurements}

An Applied Photophysics Model SP 7X nanosecond fluorescence spectrometer was used. A time-correlated single photon counting techniques was applied to the study of time-resolved emission spectra and fluorescence decay of the samples. Operation conditions were as follows: The $\mathrm{N}_{2}$ pulse lamp was operated at a $\mathrm{N}_{2}$ pressure of $c a .0 .5 \mathrm{~atm} ., 7 \mathrm{kV}$ and $80 \mathrm{kHz}$. The half-width of the lamp pulse was 7-9 ns. A PM Model 2233B photomultiplier with a cooler $\left(-5^{\circ} \mathrm{C}\right)$ was used as detector, total dynode voltage applied being $2.3 \mathrm{kV}$. In order to reduce self-absorption of the fluorescence emission a geometry of $90^{\circ}$ fluorescence from a thin layer of the solution close to the wall of the cell was used. For the neat polymer the surface fluorescence at $45^{\circ}$ incident angle to the polymer surface was observed.

Time-resolved spectra were directly recorded with the zero time taken at the instant when the lamp pulse attained $3 \%$ of its maximum pulse height. The wavelength window width of each channel was $0.5 \mathrm{~nm}$. The band width of the excitation monochromator was $10 \mathrm{~nm}$ for $\lambda 337 \mathrm{~nm}$ excitation and $5 \mathrm{~nm}$ for $\lambda 381 \mathrm{~nm}$ excitation. The band width of the emission monochromator was $5 \mathrm{~nm}$. In order to reduce the influence of pulse intensity fluctuations on the emission spectrum, fast wavelength scanning and multiple accumulation were applied to record the time-resolved emission spectra. Emission spectra of delay times longer than 150 ns were the accumulated profiles with the background deducted.

The fluorescence decay function sought was obtained by deconvolution of the directly recorded profile. A goodness of fit of the deconvolution was assessed by the randomness of the distribution of the residuals $R_{i}$ and the magnitude of the average weighted residuals $W$ defined by

$$
\begin{aligned}
W & =\frac{1}{N} \sum_{i=1}^{N} R_{i} \\
R_{i} & =\left(H_{i}-H_{i c}\right) / H_{i}^{1 / 2}
\end{aligned}
$$

where $H_{i}$ is the recorded count number of the 
$i$-th channel and $H_{i c}$ is the value calculated from the convolution of the decay function and an experimentally determined instrument response function involving the lamp pulse profile and the photomultiplier response. ${ }^{18}$

For the fluorescence decay the time width of a channel was $0.094 \mathrm{~ns}$ for most cases and $0.188 \mathrm{~ns}$ for slow decays. The count number in the channel of maximum population was (2$4) \times 10^{3}$. The condition of the excitation monochromator was the same as described above for time-resolved spectra. The band widths of the emission monochromator were 5 and $10 \mathrm{~nm}$ for the monomer and excimer bands, respectively. Excitation wavelength of 337 and $381 \mathrm{~nm}$ were used.

All measurements were done at room temperature unless otherwise stated.

\section{Steady State Fluorescence Measurement}

Steady state emission spectra of dilute solutions were recorded on a Hitachi Model MPF-4 Fluorescence Spectrophotometer for the surface fluorescence, the exciting light beam being at $60^{\circ}$ to the surface normal. The band widths of the excitation and emission monochromaters were 6 and $3 \mathrm{~nm}$, respectively.

\section{RESULTS AND DISCUSSION}

Excimer Interaction in the Concentrated Solution of DMN

The time-resolved emission spectra of a

concentrated solution $(0.115 \mathrm{M})$ of $\mathrm{DMN}$ in $\mathrm{CHCl}_{3}$ are shown in Figure 1. The intensity ratio of the longer wavelength component in the emission spectrum to the shorter wavelength one was observed to be increasing with delay time, indicating two fluorescence emitting species in the solution. The positions and the appearance of the three peaks observed at the shorter wavelength side in the time-resolved spectra are identical with those observed in steady state emission from the dilute solution of $\mathrm{DMN}{ }^{16}$ The longer wavelength peak in the late gated emission spectra is located at $c a . \lambda 425 \mathrm{~nm}$, being identical with the excimer peak in the steady state emission. ${ }^{16}$ The lifetime of excimer fluorescence is usually longer than that of the monomer fluorescence, consequently the intensity ratio of the longer wavelength emission to the shorter wavelength one is expected to increase with delay time and the $\lambda 425 \mathrm{~nm}$ emission band should be attributed to excimer emission. It is interesting to note that the monomer emission remained evident even in late gated spectra and the intensity ratio $I_{425} / I_{380}$ approached a constant value at long delay times. This is similar to the case observed by Ghiggino et al. ${ }^{19}$ in the solution of poly(1-vinyl naphthalene). This suggests the possible existence of excimer dissociation.

The fluorescence decay of the concentrated DMN solution was recorded at the monomer band, $\lambda 380 \mathrm{~nm}$, and the excimer band at
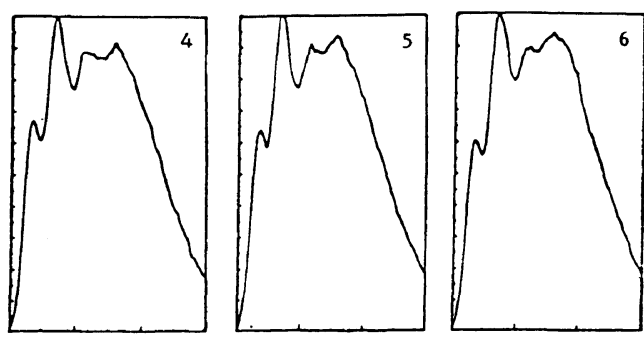

Figure 1. Time-resolved emission spectra at room temperature of a concentrated solution of DMN in $\mathrm{CHCl}_{3}(0.115 \mathrm{M})$. Gate width $5 \mathrm{~ns}$. Spectra normalized at $\lambda 380 \mathrm{~nm} .1,2,3,4,5,6$-Delay time 0, 10, 20, 30, 40, and $50 \mathrm{~ns}$, respectively. 
$\lambda 470 \mathrm{~nm}$ to avoid band overlap. Both decays were found to be non-single exponential ones. The former could be characterized as a sum of two exponential terms while the latter as a difference of two exponential terms as follows:

$$
\begin{aligned}
I_{380}= & 0.46_{3} \exp \left(-t / 1.4_{4}\right) \\
& +0.047_{4} \exp \left(-t / 15_{.0}\right), \quad W=0.91 \\
I_{470}= & -0.058_{0} \exp \left(-t / 1.2_{1}\right) \\
& +0.17_{8} \exp \left(-t / 15_{.5}\right), \quad W=1.05
\end{aligned}
$$

The double exponential character of the monomer band decay and the lifetime of the longer living component in its decay being identical within experimental error with that of the excimer band decay confirm the existence of excimer dissociation. The pre-exponential coefficient of the shorter lifetime term in the excimer band decay is negative and the lifetime value is close to that of the shorter living component in the monomer decay. These facts suggest that the excimer formation comes from the excitation of the monomer chromophores.

The fluorescence decay function for a dilute solution $\left(<10^{-3} \mathrm{M}\right)$ of DMN was found to be different from that for the concentrated solution described above. In this case a single exponential decay is sufficient. The lifetime found at various emission wavelengths was identical, being $c a$. $8 \mathrm{~ns}$, which indicates that only the excited monomers were emitting the fluorescence and excimer interaction could be ignored in dilute solutions in conformity with the observation of steady state emission.

\section{Intra-Chain Excimer Interaction between Remote Chromophores in Dilute Solutions of PTMN}

The steady state emission spectrum of a dilute solution $\left(10^{-4} \mathrm{M}\right.$ in chromophores) of the polymer PTMN in $\mathrm{CHCl}_{3}$ as shown in Figure 2 is similar to that of a dilute solution $\left(10^{-4} \mathrm{M}\right)$ of DMN. The peaks of the monomer

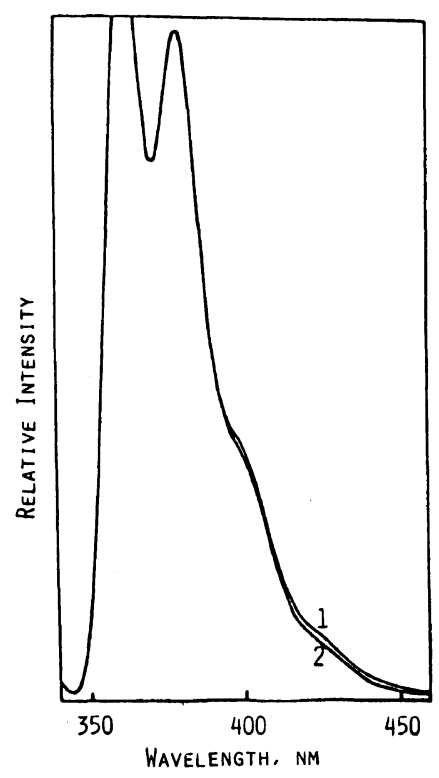

Figure 2. Steady state emission spectra at room temperature of dilute solutions $\left(10^{-4} \mathrm{M}\right)$ of 1-PTMN, 2DMN both in $\mathrm{CHCl}_{3}$.
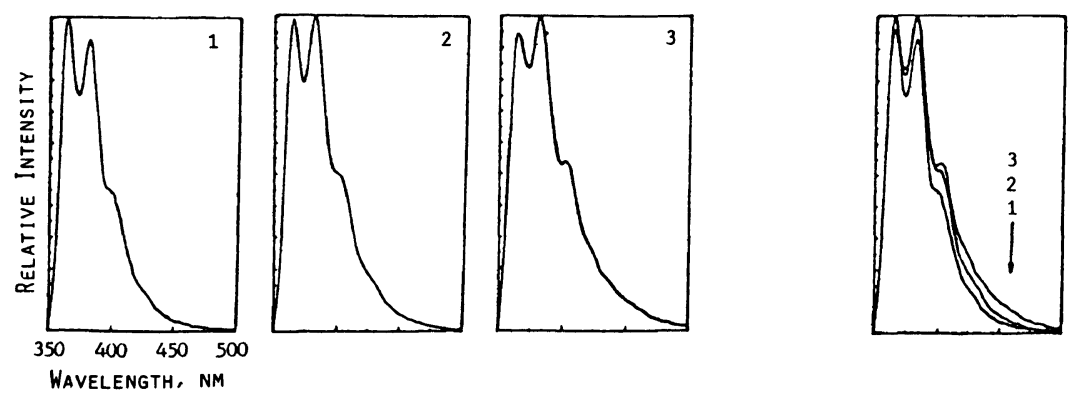

Figure 3. Time-resolved emission spectra at room temperature of a dilute solution of DTMN in $\mathrm{CHCl}_{3}$ $\left(1.25 \times 10^{-5} \mathrm{M}\right.$ in chromophores) normalized at the highest peak in the monomer emission. Gate width $5 \mathrm{~ns}$. 1,2,3-Delay time 0,25 , and $45 \mathrm{~ns}$, respectively. 
bands are located at $\lambda 365,378$, and 402 (sh) $\mathrm{nm}$. In closer inspection the former spectrum has a small tail in the long wavelength region as a polymer effect. The fluorescence decay of the monomer band from a dilute solution $\left(1.27 \times 10^{-5} \mathrm{M}\right)$ of PTMN, being different from that for a dilute DMN solution, can only be characterized by a sum of two exponential terms:

$$
\begin{aligned}
I_{380}= & 0.15_{5} \exp \left(-t / 3.6_{4}\right) \\
& +1.10_{1} \exp \left(-t / 10_{.2}\right), \quad W=1.03
\end{aligned}
$$

The time-resolved spectra of the dilute polymer solution are shown in Figure 3. The intensity ratio $I_{423} / I_{365}$ increased with delay time, being $0.15,0.19$, and 0.25 for $0-5,20-$ 25 , and $40-50 \mathrm{~ns}$ delay times, respectively. The behavior is similar to the decay functions and the time-resolved emission spectra for a concentrated DMN solution. So the longer wavelength component of the fluorescence from the dilute solution of PTMN is attributed to an excimer emission. However, this excimer interaction could not be of inter-chain origin since the concentration is one order of magnitude smaller than the critical concentration, $1.45 \times 10^{-4} \mathrm{M}$, below which the ratio of excimer to monomer intensity in steady state fluorescence remains constant independent of concentration. ${ }^{16}$ Consequently this excimer interaction comes from intra-chain chromophores which are at least separated by a

polyether soft segment of molar mass $1.66 \times$ $10^{3}$. These chromophores, however, might spatially close to each other by back coiling of the very flexible polyether moiety of the polymer chain to form the excimer site. Similar intrachain excimer fluorescence has been reported for polymers containing naphthalene groups in the main chain separated by flexible spacers. ${ }^{14,15}$

For a dilute solution of PTMN $(1.25 \times$ $\left.10^{-5} \mathrm{M}\right)$ in a mixed solvent, $\mathrm{CH}_{3} \mathrm{OH}$ : $\mathrm{CHCl}_{3}=2.5: 1 \mathrm{v} / \mathrm{v}$, the fluorescence decay of the monomer band follows the two exponential term function:

$$
\begin{aligned}
I_{380}= & 0.18_{9} \exp \left(-t / 2.5_{8}\right) \\
& +0.11_{0} \exp \left(-t / 9.0_{9}\right), \quad W=0.97
\end{aligned}
$$

The time-resolved emission spectra of the solution are shown in Figure 4. This is typical of the effect of mixed solvent on intra-chain excimer formation between remote chromophores on a flexible polymer chain. ${ }^{14,15}$ As $\mathrm{CH}_{3} \mathrm{OH}$ is a non-solvent for PTMN the polymer coil dimension shrinks in the mixed solvent to give a higher spatial segment density leading to a higher probability of intra-chain excimer formation.

\section{Excimer Interaction in Concentrated Solution of PTMN}

The time-resolved emission spectra of a concentrated solution $\left(1.31 \times 10^{-2} \mathrm{M}\right)$ of
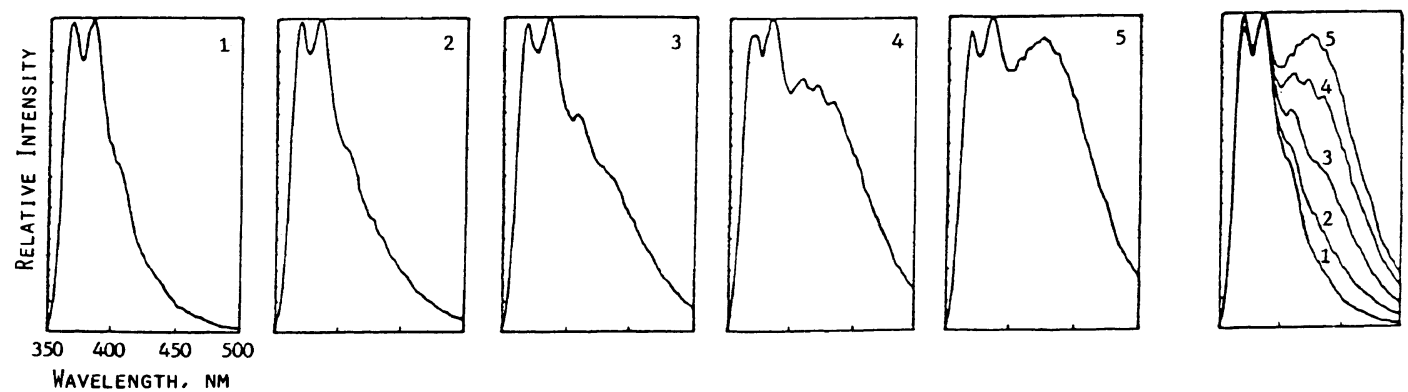

Figure 4. Time-resolved emission spectra at room temperature of a dilute solution of PTMN $\left(1.25 \times 10^{-5} \mathrm{M}\right)$ in a mixed solvent, $\mathrm{CH}_{3} \mathrm{OH}: \mathrm{CHCl}_{3}=2.5: 1 \mathrm{v} / \mathrm{v}$, normalized at $\lambda 380 \mathrm{~nm}$. Gate width $5 \mathrm{~ns}$. $1,2,3,4,5$-Delay time $0,20,30,40$, and $50 \mathrm{~ns}$, respectively. 

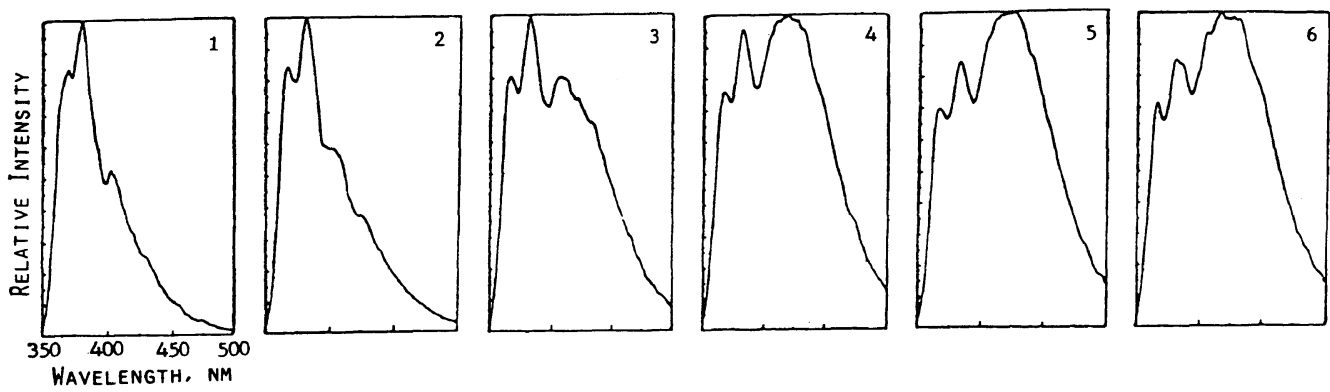

Figure 5. Time-resolved emission spectra at room temperature of a concentrated solution of PTMN in $\mathrm{CHCl}_{3}(0.235 \mathrm{~g} / \mathrm{ml})$ normalized at the highest emission peak. Gate width $5 \mathrm{~ns}$. 1,2,3,4,5,6-Delay time 0, 10, $20,30,40$, and $50 \mathrm{~ns}$, respectively.

PTMN are shown in Figure 5. The intensity ratio $I_{\mathrm{E}} / I_{\mathrm{M}}$ of the excimer to monomer fluorescence increased with delay time, similar to but more pronounced than that of a concentrated solution of DMN at the same chromophore concentration. After $40 \mathrm{~ns}$ delay time $I_{\mathrm{E}} / I_{\mathrm{M}}$ approached a constant, indicating dissociation of the excimer. The peak position in the late gated spectra is located at $\lambda 420-430 \mathrm{~nm}$, identical with the excimer peak in the steady state emission spectrum. ${ }^{16}$ The concentration of the solution far exceeds the concentration $\mathrm{c}^{+}$where the spatial segment density becomes approximately uniform throughout the solution, both intra- and inter-chain excimer interactions prevail in the solution.

The decays of the monomer and the excimer bands can only be fitted by a sum and a difference of two exponential terms, respectively:

$$
\begin{aligned}
I_{380}= & 0.32_{6} \exp \left(-t / 2.3_{5}\right) \\
& +0.050_{4} \exp \left(-t / 12_{.3}\right), \quad W=0.97 \\
I_{450}= & -0.10_{9} \exp \left(-t / 2.7_{1}\right) \\
& +0.20_{7} \exp \left(-t / 12_{.6}\right), \quad W=1.03
\end{aligned}
$$

The shorter and longer lifetimes of the decay components of the monomer band are identical with those of the excimer band decay within experimental error, similar to the results obtained from the concentrated solution of DMN.

\section{Excimer Interaction in the Neat Polymer}

PTMN polymer film when cast from solution was amorphous. On standing the polyether soft segments crystallized which showed a melting point around $30^{\circ} \mathrm{C}$ and the naphthalene dicarboxyloyl moieties of the polymer crystallized slowly in the course of weeeks to months at room temperature. ${ }^{16,17}$ It was found that a PTMN film which had been laid aside for $25 \mathrm{~d}$ showed in its excitation spectrum for excimer emission a shoulder at the long wavelength side of the peak at $\lambda 362 \mathrm{~nm}$. The film after $285 \mathrm{~d}$ showed a pronounced peak at $\lambda 379 \mathrm{~nm}$ with a height higher than the peak at $\lambda 362 \mathrm{~nm}$ in the excitation spectrum for excimer emission. ${ }^{16,20}$ Heating the crystallized film under $\mathrm{N}_{2}$ to $c a .200^{\circ} \mathrm{C}$ for $20 \mathrm{~min}$ and then cooling down to room temperature reduced greatly the height of the second excitation peak at $\lambda 379 \mathrm{~nm}$. These facts indicate that crystallization of the naphthalene dicarboxyloyl moieties of the PTMN chains originates from stacking of the planar aromatic ring chromophores which act at the same time as preformed excimer sites.

The time-resolved emission spectra with $\lambda 381 \mathrm{~nm}$ excitation are shown in Figure 6. In the $0-5 \mathrm{~ns}$ emission spectrum the excimer peak at $\lambda 423 \mathrm{~nm}$ appeared already and in addition a smaller peak appeared at $i 400 \mathrm{~nm}$ which submerged very quickly with delay time. The fluorescence decay is of double exponential term character with a shorter lifetime of 


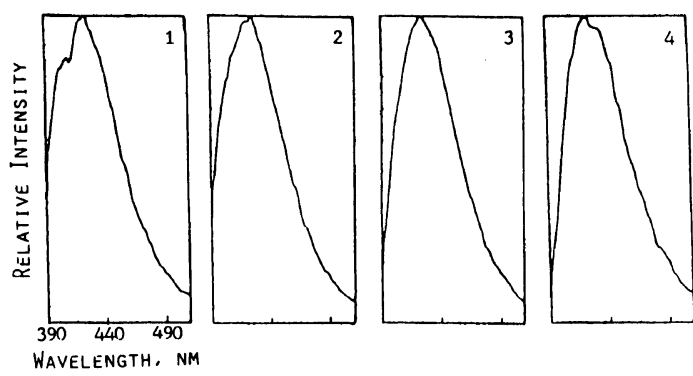

Figure 6. Time-resolved emission spectra at room temperature of the neat polymer PTMN with $\lambda 381 \mathrm{~nm}$ excitation, normalized at $\lambda 423 \mathrm{~nm}$. Gate width $5 \mathrm{~ns}$. 1,2,3,4-Delay time $0,5,10$, and $50 \mathrm{~ns}$, respectively.

$3 \mathrm{~ns}$ and a longer lifetime of $9 \mathrm{~ns}$. The $\lambda 400 \mathrm{~nm}$ peak in the early gated spectra and the shorter lifetime term in the decay function should be attributed to the monomer fluorescence. However, the photon energy of $\lambda 381 \mathrm{~nm}$ incident radiation is less than that of the $0-0$ band at $\lambda 362 \mathrm{~nm}$. It is obvious that a possible explanation would be a hot band excitation of the chromophore not belonging to the excimer site, i.e., the excitation of the chromophore not in its vibrational ground state but rather in a vibrational excited state of the electronic ground state to the first excited siglet state. This view is substantiated by observation of the polymer at liquid nitrogen temperature, whence the rubbery polymer at room temperature became a transparent glass. When excited with $\lambda 381 \mathrm{~nm}$ radiation no short wavelength component appeared in the early gated spectra as shown in the time-resolved emission spectra shown in Figure 7 . Then the decays at $i 423$, 450 , and $470 \mathrm{~nm}$ emission can all be characterized by a single exponential term of lifetime $9.2 \mathrm{~ns}$ with good fit shown in Figure 8. The chromophores at liquid nitrogen temperature are surely in their vibrational ground state and no hot band excitation is possible. This also demonstrates clearly the direct excitation of the excimer fluorescence from stacked naphthalene dicarboxyloyl groups as preformed excimer sites during long standing in the rubbery state at room temperature and they are

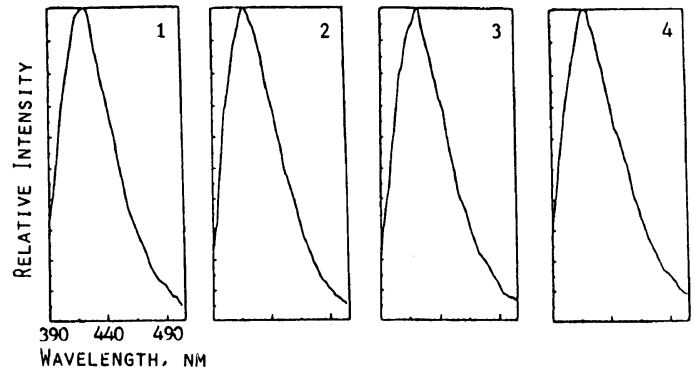

Figure 7. Time-resolved emission spectra at $77 \mathrm{~K}$ of the neat polymer PTMN with $\lambda 381 \mathrm{~nm}$ excitation, normalized at $\lambda 423 \mathrm{~nm}$. Gate width $10 \mathrm{~ns}$ for spectra 1,2 and 3. 1,2,3,4-Delay time $0,10,20 \mathrm{~ns}$, and $0-100 \mathrm{~ns}$, respectively.
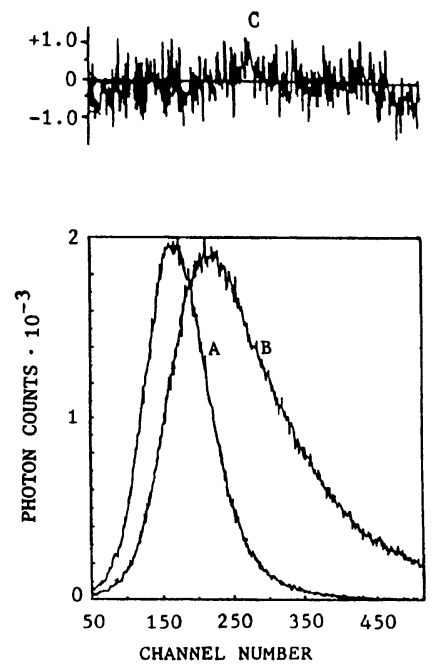

Figure 8. Single exponential decay at room temperature of the excimer band directly excited from the stacked chromophores. A, the lamp pulse at the excitation wavelength $381 \mathrm{~nm}$; B, the decay profile at $\lambda 450 \mathrm{~nm}$; C, distribution of the weighted residuals between the decay profile and deconvoluted decay function with the lamp pulse. The deconvoluted decay function, $\exp (-t / 9.194)$.

frozen into the glass state at $77 \mathrm{~K}$, as no segmental motion of the polyether soft segments in the polymer is possible at this temperature. It is interesting to point out in passing that in the previously published steady state excimer spectra of the polymer excited by $\lambda 370$ and by $\lambda 380 \mathrm{~nm}$ radiation ${ }^{16,20}$ a monomer emission was also present as a shoulder 

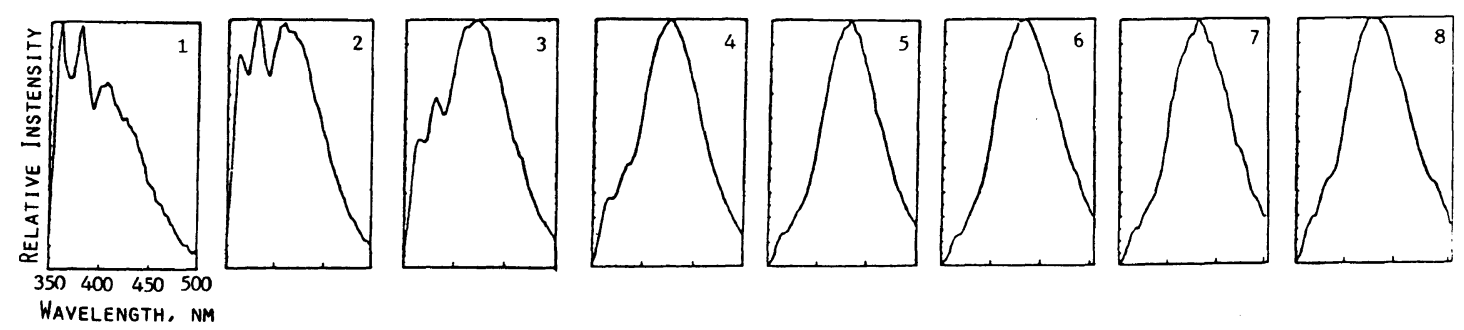

Figure 9. Time-resolved emission spectra at room temperature of the neat polymer PTMN with $\lambda 337 \mathrm{~nm}$ excitation. Gate width $5 \mathrm{~ns}$ for spectra $1-4,10 \mathrm{~ns}$ for spectrum $5,20 \mathrm{~ns}$ for spectrum 6 and $50 \mathrm{~ns}$ for spectra 7, 8. 1,2,3,4,5,6,7,8-Delay time $0,10,15,20,40,60,100$, and $150 \mathrm{~ns}$.

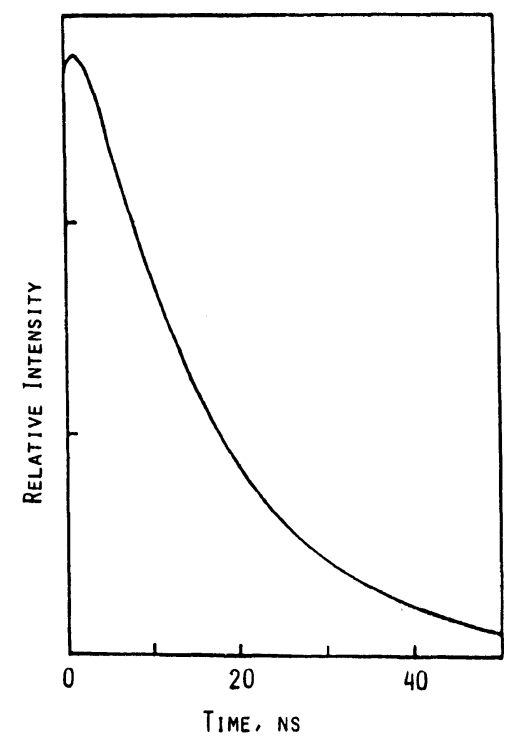

Figure 10. Deconvoluted fluorescence decay at room temperature of the excimer band $(\lambda 450 \mathrm{~nm})$ from the neat polymer PTMN with $\lambda 337 \mathrm{~nm}$ excitation.

around $\lambda 400 \mathrm{~nm}$ due to overlapping with the directly excited excimer band.

With $\lambda 337 \mathrm{~nm}$ excitation the time-resolved emission spectra are shown in Figure 9. The three monomer fluorescence peaks in the early gated emission spectra were submerged fastly by the excimer band in $40 \mathrm{~ns}$ delay time. However the relative intensity of the monomer to the excimer emission increased again after $150 \mathrm{~ns}$ dealy time. The nature of this abnormal phenomenon is not clear at the present moment.

The dacays of the monomer and the excimer bands of the neat polymer excited by $\lambda 337 \mathrm{~nm}$ radiation at room temperature could only be fitted by a sum and a difference of two exponential terms respectively.

$$
\begin{aligned}
I_{380}= & 0.49_{3} \exp \left(-t / 2.0_{0}\right) \\
& +0.033_{3} \exp \left(-t / 14_{.4}\right), \quad W=1.11 \\
I_{450}= & -0.034_{0} \exp \left(-t / 1.6_{6}\right) \\
& +0.17_{0} \exp \left(-t / 14_{.5}\right), \quad W=1.09
\end{aligned}
$$

In the excimer fluorescence decay functions with $\lambda 337 \mathrm{~nm}$ excitation the ratios of the preexponential coefficients of the shorter lifetime term to that of the longer lifetime term are found to be $-1 / 3,-1 / 2$, and $-1 / 5$ for the concentrated solutions of DMN and PTMN and the neat polymer respectively. These values are different from the value -1 expected from Birk's diffusion controlled mechanism. ${ }^{21}$ This might be the consequence of the existence of preformed stacked chromophores as excimer sites and their direct excitation in addition to the diffusion mechanism of excimer formation.

The deconvoluted decay curve of the excimer band of the neat polymer excited by $\lambda 337 \mathrm{~nm}$ radiation is shown in Figure 10. The excimer emission intensity did not start from zero at the zero time but from a rather high value close a maximum reached within the delay time of $1 \mathrm{~ns}$ and then decayed. This is again a direct evidence of the direct excitation of preformed excimer sites in the polymer. 


\section{Excimer Fluorescence of PTMN}

\section{REFERENCES}

1. S. S. Yanari, F. A. Bovey, and R. Lumry, Nature, 200, 242 (1963).

2. T. Ishii, T. Handa, and S. Matsunaga, Macromolecules, 11, 40 (1978).

3. C. David, M. Piens, and G. Geuskens, Eur. Polym. J., 8, 1019 (1972).

4. W. Klöpfer, J. Chem. Phys., 50, 2337 (1969).

5. A. C. Somersall and J. E. Guillet, Macromolecules, 6, 218 (1973).

6. M. Yokoyama, T. Tamamura, M. Atsumi, M. Yoshimura, Y. Shirota, and H. Mikawa, Macromolecules, 8, 101 (1975).

7. R. B. Fox, T. R. Price, R. F. Cozzens, and J. R. McDonald, J. Chem. Phys., 57, 534 (1972).

8. I. Soutar, D. Phillips, A. J. Roberts, and G Rumbles, J. Polym. Sci. Polym. Phys. Ed., 20, 1759 (1982).

9. D. Phillips, A. J. Roberts, G. Rumbles, and I. Soutar, Macromolecules, 16, 1597 (1983).

10. D. Phillips, A. J. Roberts, and I. Soutar, J. Polym.
Sci., Polym. Phys. Ed., 18, 2401 (1980).

11. D. Phillips, A. J. Roberts, and I. Soutar, J. Polym. Sci., Polym. Phys. Ed., 18, 123 (1980).

12. S. Ito, M. Yamamoto, and Y. Nishijima, Polym. J., 13, 791 (1981).

13. W. E. Lindsell, F. C. Robertson, and I. Soutar, Eur. Polym. J., 17, 791 (1981).

14. J. A. Ibemesi, J. B. Kisinger, and M. A. El-Bayoumi, J. Polym. Sci., Polym. Chem. Ed., 18, 879 (1980).

15. C. E. Hoyle and K. J. Kim, Macromolecules, 20, 597 (1987).

16. T. Cao and R. Qian, Acta Chim. Sin., 42, 51 (1984).

17. T. Cao and R. Qian, New Polym. Mater., 1, 87 (1988).

18. D. Hu, X. Zhang, R. Ma, and C. Guo, Spectrosc. Spect. Anal. (China), 2, 163 (1982).

19. K. P. Ghiggino, R. D. Wright, and D. Phillips, Chem. Phys. Lett., 53, 552 (1978).

20. R. Qian, "Macromolecules," H. Benoit and P. Rempp, Ed., Pergamon Press, Oxford, 1982, p. 139.

21. J. B. Birks and L. G. Christophoron, Proc. R. Soc. London, 275, 575 (1963). 